\title{
Aseismic Layer Detected in the Mid-Crust of Nubra-Siachen Region, India Using Local Seismic Data
}

\author{
Rajinder Parshad1,2*, Snehmani ${ }^{3}$, Pankaj Kumar Srivastva², Reeta Rani' ${ }^{3}$, \\ Ashwagosha Ganju ${ }^{3}$ \\ ${ }^{1}$ Geological Survey of India, Faridabad, India \\ ${ }^{2}$ Deptt. of Petroleum Engineering and earth sciences, UPES, Dehradun, India \\ ${ }^{3}$ Snow and Avalanche Study Establishment, Research and Development Center, Chandigarh, India \\ Email: ${ }^{*}$ sharma.dab@gmail.com
}

Received 10 July 2015; accepted 19 September 2015; published 22 September 2015

Copyright (C) 2015 by authors and Scientific Research Publishing Inc.

This work is licensed under the Creative Commons Attribution International License (CC BY). http://creativecommons.org/licenses/by/4.0/

cC) (i) Open Access

\section{Abstract}

The local seismicity observed by seismic network in siachen-nubra region during January 2010December 2012 shows that the middle part of the crust $(17-40 \mathrm{~km})$ is aseismic. This aseismic layer $(17-40 \mathrm{~km})$ is sandwiched between two seismically active layers and depicts a good spatial correlation with the observations of low resistivity reported from magnetotelluric studies for the same region. The local seismicity shows a trend along the Karakoram fault and clustering of events in Shyok Suture zone and Karakoram shear zone. The moment magnitude of these events lies between 1.3 and 4.3. Most of these events have been originated in upper crust.

\section{Keywords}

Shear Zone, Karakorum, Local Events, Aseismic, Nubra-Siachen

\section{Introduction}

The Himalayas, spanning length of $2500 \mathrm{~km}$ is a consequence of continent collision [1]-[3]. The Himalayan region is known for its high seismicity for small to large magnitude earthquakes. Five large magnitude earthquakes have visited Himalayan region (mag. > 8.4) and nine earthquakes of magnitude $>7.0$ since 1897. Beside these large magnitude earthquakes, numerous small magnitude (Magnitude < 3.0) earthquakes have visited the Himalaya.

Nubra valley locates in the eastern Karakorum region of northern Ladakh and extends from Siachen glacier

\footnotetext{
${ }^{*}$ Corresponding author.
} 
system in North-West to Shyok-Nubra confluence in South East. It covers $4381.23 \mathrm{sq} \mathrm{km}$ area and amassed of various litho units such as Karakorum plutonic complex, Ladakh Plutonic Complex, etc. Siachen Glacier is more than a valley glacier; it is a system of glaciers, consisting of Northern, Central and Southern glaciers [4]. Siachen glacier along with its tributaries covers more than $1500 \mathrm{sq}$ kms. This is the largest glacier in the Himalaya and second largest among the known glacier outside the polar and sub-polar regions. Siachen lies between the Saltoro Range immediately to the west and the main Karakoram Range to the east. The melting waters of the glacier are the main source of the Nubra River, which meets the Shyok River near Khalasr forming Nubra-Shyok confluence (Figure 1). The Shyok in its onward journey joins Indus River; thus the glacier is a major source of water for the Indus.

A network of digital broadband seismograph equipped with 3-component digital seismometer (Trillium 120P) is operational in Nubra-Valley, since 2008. The seismic data recorded (Jan'10-Dec'12) at these stations have been used for this study. The hypocentral depth of the local events recorded at seismic stations is in the upper crust depth range $0-17 \mathrm{~km}$ and $40-75 \mathrm{~km}$ but few events are observed at the depth greater than $100 \mathrm{~km}$ in the mantle along the Karakorum fault.

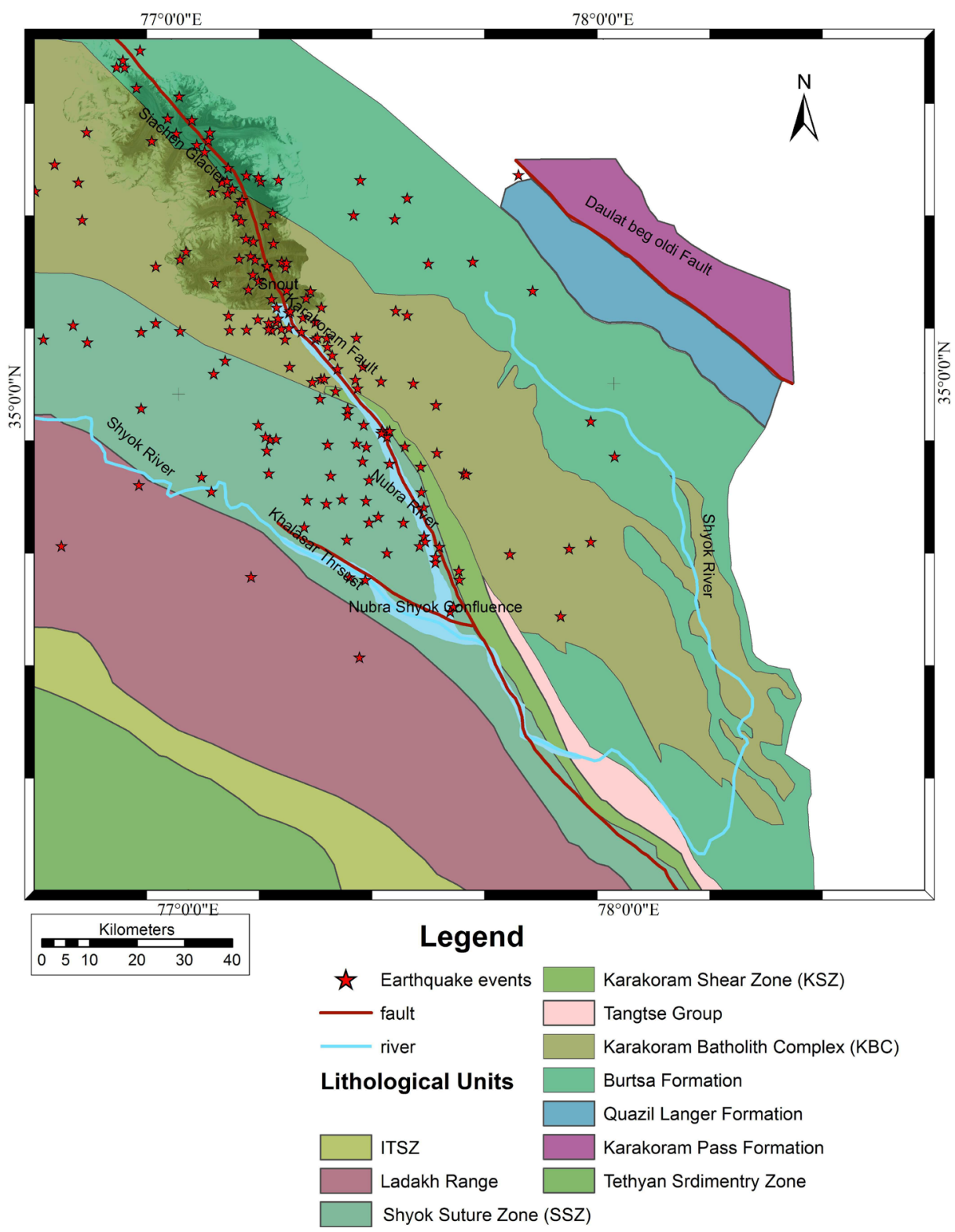

Figure 1. The earthquake events (Table 2) used in this study is shown on the Geological map [16] of the area. 


\section{Tectonic Set up of the Area}

The collision between the Eurasian plate and the Indian plate started about 55 Ma ago [5], and is still continuing at the present time [6]. This successive collision forms the Himalaya and the Earth's largest and highest plateau with anomalously thick crust. Different models have been proposed by various research workers to explain the origin of the Himalaya [2] [3] [7]. The collision has also resulted into large scale thrusting and the same is progressed southward forming the Main Central Thrust (MCT) and Main Boundary Thrust (MBT). The IndusTsangpo Suture (ITSZ) located about $250 \mathrm{~km}$. North of MCT marks the pre-collision boundary along which the Indian plate subducted below the Eurasian plate and the subduction ended in Eocene times [7]. Along the MCT, the Higher Himalaya overthrusted the Lesser Himalaya along MBT, the Lesser Himalaya thrusted over the Siwaliks.

The crustal thickness using, 15 - 60 s Rayleigh wave group velocities with receiver functions from teleseismic arrivals was mapped up to $75 \mathrm{~km}$ at Taksha station in Nubra region [8]. Nubra region is tectonically highly disturbed [9] and this region is bisected by Karakoram fault [10]-[13]. Approximately $800 \mathrm{~km}$, dextral strike slip Karakoram fault is the prominent tectonic feature in the region [14] [15], this fault runs almost parallel to the main body of Siachen glacier. The other major tectonic features of this region are Karakoram shear zone, Shyok Suture zone, Khalsar thrust, etc. Karakoram Shear Zone (KSZ) is intensely mylonitized granite gneiss, volcanic, conglomerate, slate-phyllite-limestone intercalations, amphibolites and serpentinite intervene the Shyok Suture Zone and the frontal Asian Plate margin along the Nubra-Shyok Valleys for nearly $200 \mathrm{~km}$ to form the $1-5 \mathrm{~km}$ wide KSZ [16].

This region falls in the zone IV of the seismic zoning map of India [17], and seismotectonic atlas of India and its enviors [18]. Geographical position of study region is shown in Figure 2, and this region is tectonically active due to the presence of Karakoram fault and various structural features like Shyok Suture Zone (SSZ), Karakorum Shear Zone (KSZ). This region is almost virgin as far as local seismological observations are concerned, prior to the installation of this network. This region is remote and accessible through world highest motor able

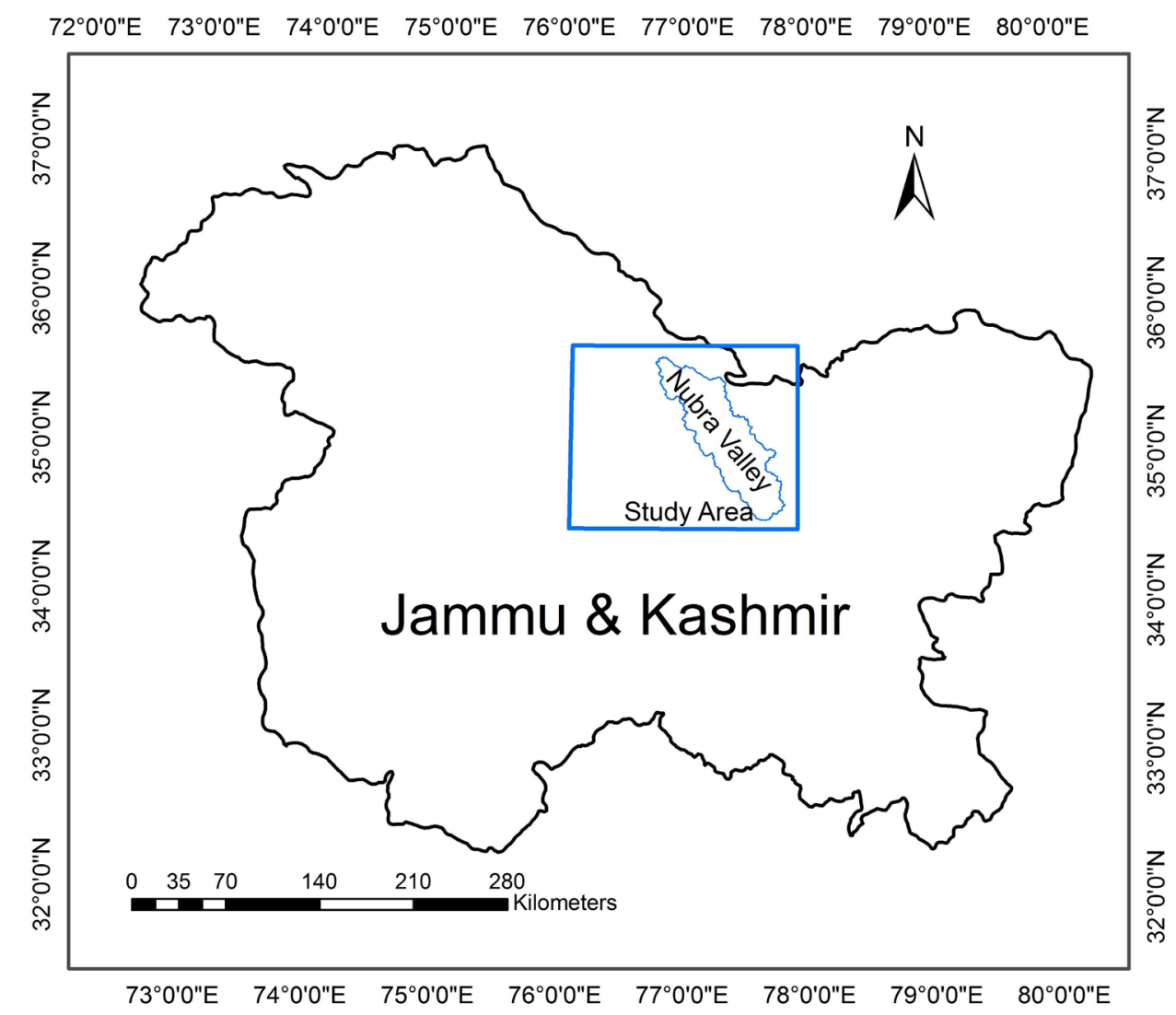

Figure 2. Geographical position of study area. 
pass Khardung La (18,380 feet) for a very limited period in the year. Owing to remoteness and harsh climatic condition throughout the year, maintenance and operation of seismic network is a big challenge in nubra-siachen region. However four, broadband seismometers has been installed during 2008 in this region. This region did not experience any major earthquake till date (as per the literature/record available). However micro, small \& large earthquakes frequently experienced by local residing in this region [19] [20]. The local seismicity observed by this seismic network is mainly mapped along the Karakoram Fault, Karakoram Shear zone \& Shyok Suture zone.

\section{Data Used and Methodology}

The local seismological network comprising of four broad band recording station has been installed in Nubra Valley region, Jammu \& Kashmir. The layout of the network is shown in Figure 3 and the details of recording stations are given in Table 1. The four recording stations used the same type of instrumentation i.e. the Trillium $120 \mathrm{P}$ broadband seismometer and Taurus 24 bit data acquisition system, manufactured by M/s Nanometrics, these instruments are operational in continuous mode. The seismic data recorded at Nubra valley seismological network and used for present analysis is given in Table 2. The 3-C seismogram of one of the recorded event are shown in Figure 4. Data were recorded with a sampling rate of 100 samples per second. For computation of hypocentral parameters of Local earthquakes three types of data is required such as phase data of local earthquake, accurate geographical co-ordinates along with elevation of recording stations and the local velocity model of the region covered by the recording stations. Additionally a computinally efficient and stable computer program is a major requirement to allow conversion of travel time data to estimate hypocentral parameters [21] [22]. The seismic velocity model determined by [23] at Taksha seismic station (TKS) has been used for estimation of hypocentral parameters of local earthquakes. The "HYPCENTER 3.2" given by [24] and incorporated in SEISAN environment has been used for the estimation of hypocentral parameters of local earthquakes. The moment magnitude (MW) has been estimated using [25] given as below.

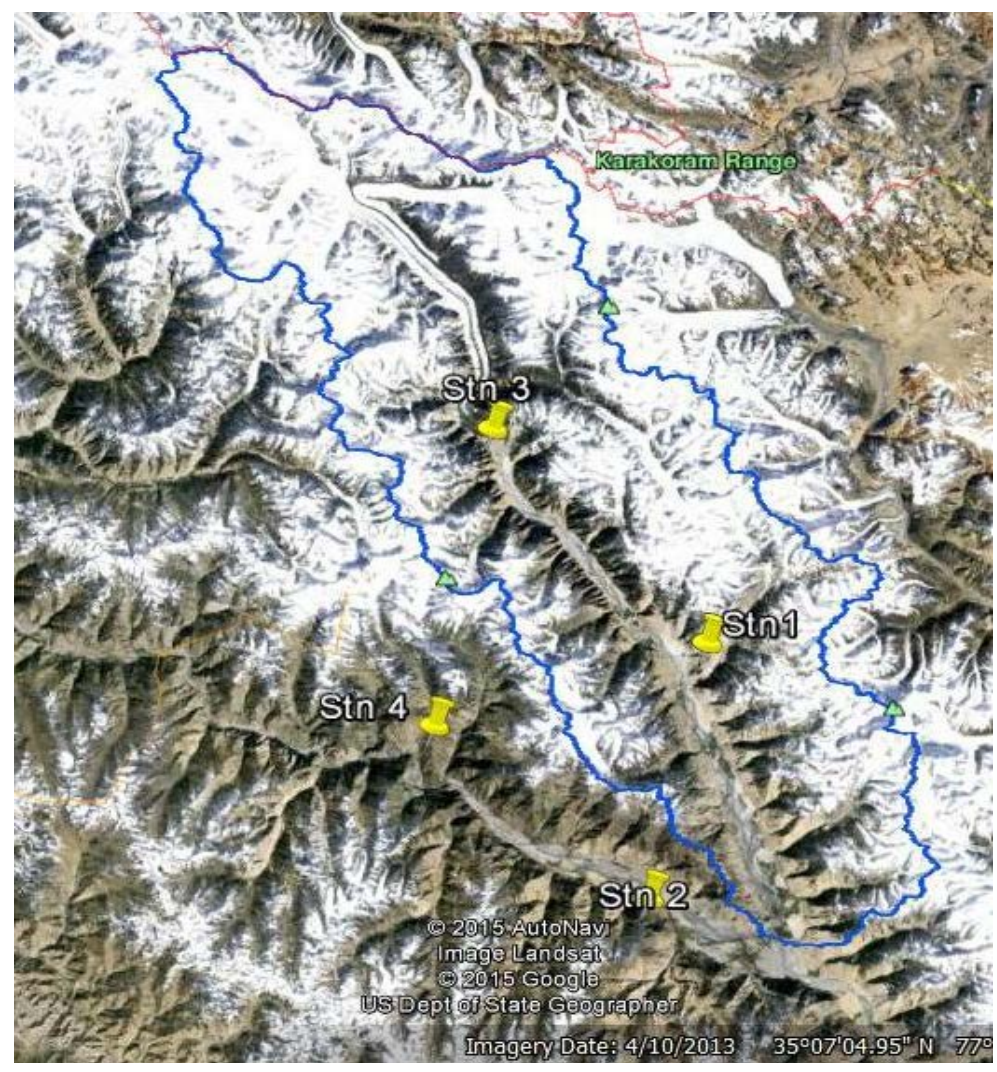

Figure 3. Layout plan of local seismic stations of Nubra-Siachen region shown on Google earth image. 

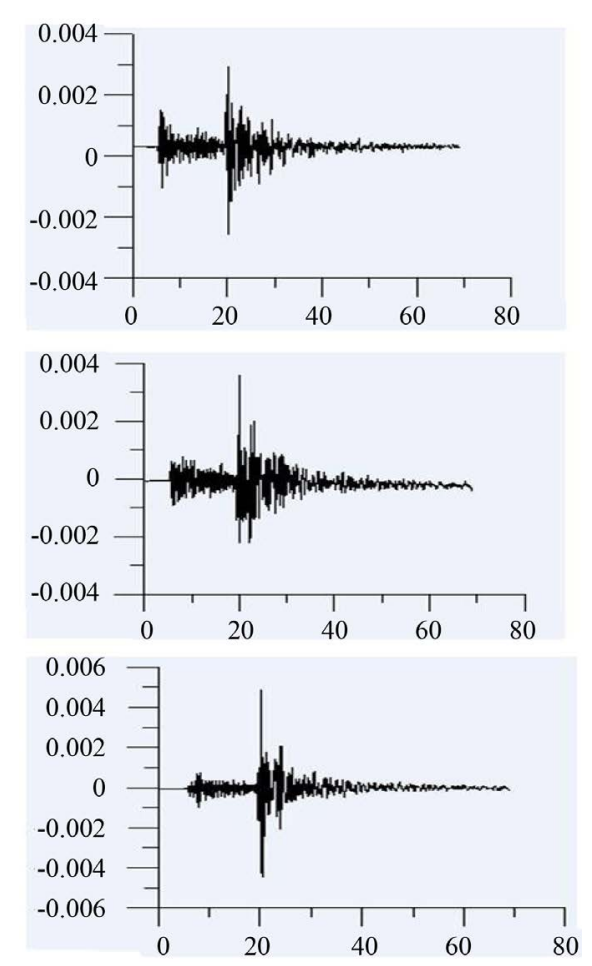

Figure 4. 3-C Seismogram of Local earthquake recorded at seismic network in Nubra-Siachen region (Z, N-S, E-W from top to bottom).

Table 1. Details of seismic station installed in area of study.

\begin{tabular}{cccccc}
\hline Sr.No. & Station Code & $\begin{array}{c}\text { Latitude } \\
\text { (Degree) }\end{array}$ & $\begin{array}{c}\text { Longitude } \\
\text { (Degree) }\end{array}$ & $\begin{array}{c}\text { Elevation w.r.t. Mean Sea Level } \\
\text { (Meters) }\end{array}$ & $\begin{array}{c}\text { Mode of } \\
\text { Operation }\end{array}$ \\
\hline 1 & Stn 1 & $77.49 \mathrm{E}$ & $34.92 \mathrm{~N}$ & 3430 & Digital \\
2 & Stn 2 & $77.41 \mathrm{E}$ & $34.61 \mathrm{~N}$ & 3180 & Digital \\
3 & Stn 3 & $77.2 \mathrm{E}$ & $35.18 \mathrm{~N}$ & 3455 & Digital \\
4 & Stn 4 & $77.12 \mathrm{E}$ & $34.82 \mathrm{~N}$ & 3180 & Digital \\
\hline
\end{tabular}

$$
M w=\frac{\log M_{0}}{1.5}-10.7
$$

\section{Result and Discussions}

The hypocentral parameters of local earthquake are estimated with the help of "HYPOCENTER 3.2" [24] from the phase data recorded at seismic stations. The standard error in the estimation of hypocentral parameters for these events are $<0.5$ seconds in origin time, $<5.0 \mathrm{Km}$ in epicenter (ERH) and $<6.0$ in focal depth (ERZ).

The local seismicity is mainly oriented with the Karakoram Fault and clustered in Shyok Suture Zone and Karakoram shear zone. The depth trend of earthquake events (Figure 5) shows that the seismicity in the Siachen region is mainly occurring at upper crustal depth. However few events are occurred at a depth of $40 \mathrm{~km}$ and more but no local event is reported in a depth range from 17 - $40 \mathrm{~km}$ below the surface of the earth. This seismically inert layer (17 - $40 \mathrm{~km}$ ) shows that no stress get accumulated in this layer. This layer shows good spatial correlation with the low resistivity layer in the mid crust of NW Himalaya determined from the magnetotelluric studies of NW Himalaya [26] \& Partially melted upper middle crust revealed by Rayleigh wave dispersion [23]. Due to the partially melted nature of mid-crust (depth range $17-40 \mathrm{~km}$ ) no stress is accumulated in the midllecrust, so no earthquake is reported in mid-crustal depth range in Siachen region. This infers the presence of aseismic layer in the mid-crust part. This aseismic layer is sandwiched between seismically active layers in Siachen 
Table 2. List of Local events recorded at Nubra-Siachen seismic Network.

\begin{tabular}{|c|c|c|c|c|c|c|c|c|c|}
\hline \multirow[b]{2}{*}{ Sr No. } & \multicolumn{2}{|c|}{ Origin Time } & \multicolumn{3}{|c|}{ Hypocentral Parameters } & \multirow[b]{2}{*}{ MW } & \multirow[b]{2}{*}{ RMS } & \multirow[b]{2}{*}{$\begin{array}{l}\text { ERH } \\
(\mathbf{K m})\end{array}$} & \multirow[b]{2}{*}{$\begin{array}{l}\text { ERZ } \\
(\mathbf{K m}\end{array}$} \\
\hline & DD-MM-YYYY & HR:MIN:SEC & Longitude & Latitude & $\begin{array}{c}\text { Depth } \\
\text { (km) }\end{array}$ & & & & \\
\hline 1 & 01-01-2010 & 10:19:57 & 77.37 & 35.828 & 10 & 2.5 & 0.4 & 4.7 & 3.7 \\
\hline 2 & 03-01-2010 & 04:06:09 & 77.466 & 34.923 & 53.2 & 3.2 & 0.21 & 5 & 1.9 \\
\hline 3 & 08-01-2010 & $05: 45: 42$ & 77.16 & 35.12 & 10 & 3.7 & 0.36 & 3.2 & 2.7 \\
\hline 4 & $14-01-2010$ & 08:32:09 & 77.419 & 34.866 & 0.1 & 2.5 & 0.47 & 4.3 & 1.2 \\
\hline 5 & $14-01-2010$ & $17: 24: 54$ & 77.425 & 34.791 & 10 & 3.1 & 0.58 & 2.4 & 3.9 \\
\hline 6 & $17-01-2010$ & 07:09:52 & 78.832 & 34.831 & 10.3 & 2 & 0.21 & 5 & 1.9 \\
\hline 7 & 22-01-2010 & $02: 51: 40$ & 77.466 & 34.923 & 3.2 & 3 & 0.39 & 4.6 & 3.8 \\
\hline 8 & $25-01-2010$ & $22: 12: 18$ & 77.582 & 35.237 & 15.8 & 2.8 & 0.89 & 1.7 & 3.4 \\
\hline 9 & 08-02-2010 & 03:21:12 & 77.477 & 34.92 & 10 & 4.2 & 0.76 & 3.4 & 2.6 \\
\hline 10 & 09-02-2010 & $11: 26: 00$ & 76.951 & 35.136 & 10 & 3 & 0.63 & 5 & 1.7 \\
\hline 11 & $10-02-2010$ & 03:19:00 & 77.109 & 35.062 & 7.3 & 2.9 & 0.42 & 1.2 & 3.6 \\
\hline 12 & $11-02-2010$ & 01:42:00 & 77.507 & 35.323 & 10 & 2.4 & 0.53 & 4.3 & 4.9 \\
\hline 13 & $11-02-2010$ & 11:51:03 & 77.59 & 34.878 & 10.4 & 2.6 & 0.56 & 5 & 3.7 \\
\hline 14 & $14-02-2010$ & 06:09:44 & 77.651 & 34.838 & 0 & 2.8 & 0.39 & 4.8 & 1.7 \\
\hline 15 & $18-02-2010$ & 01:20:07 & 77.37 & 34.795 & 16.2 & 2.6 & 0.57 & 5.2 & 3.5 \\
\hline 16 & $25-02-2010$ & $12: 19: 50$ & 77.751 & 34.683 & 98.7 & 3.6 & 0.17 & 3.7 & 2.9 \\
\hline 17 & 26-02-2010 & 03:07:23 & 77.656 & 34.836 & 10 & 2.9 & 0.34 & 4.9 & 2.6 \\
\hline 18 & 06-03-2010 & $20: 01: 56$ & 77.182 & 34.939 & 8.6 & 1.9 & 0.12 & 4.3 & 3.8 \\
\hline 19 & $12-03-2010$ & 07:05:18 & 77.379 & 34.718 & 10 & 2.1 & 0.26 & 2.9 & 3.1 \\
\hline 20 & 21-03-2010 & 01:42:18 & 76.725 & 34.717 & 96 & 2.4 & 0.69 & 1.3 & 4.9 \\
\hline 21 & 28-03-2010 & $12: 55: 00$ & 76.905 & 34.83 & 52.8 & 2.2 & 0.34 & 3.7 & 1.9 \\
\hline 22 & $16-04-2010$ & $12: 19: 50$ & 77.044 & 35.519 & 51.2 & 2.9 & 0.43 & 2.6 & 2.1 \\
\hline 23 & 23-04-2010 & 00:28:45 & 78.735 & 33.297 & 10 & 1.8 & 0.56 & 1.8 & 2.6 \\
\hline 24 & 25-04-2010 & 20:01:56 & 77.153 & 35.326 & 54 & 3.2 & 0.71 & 2.8 & 1.9 \\
\hline 25 & 29-04-2010 & 06:43:12 & 77.654 & 33.04 & 2.7 & 2.6 & 0.63 & 4.3 & 3.2 \\
\hline 26 & 02-05-2010 & $13: 32: 13$ & 77.464 & 34.918 & 56.7 & 4.1 & 0.59 & 3.1 & 2.7 \\
\hline 27 & 06-05-2010 & $21: 51: 17$ & 77.383 & 34.647 & 68 & 2.7 & 0.89 & 1.7 & 3.9 \\
\hline 28 & 07-05-2010 & $13: 26: 00$ & 77.248 & 35.1 & 14 & 4.4 & 0.63 & 2.6 & 3.2 \\
\hline 29 & $14-05-2010$ & $02: 27: 26$ & 76.644 & 35.721 & 56.2 & 3.7 & 0.72 & 1.7 & 2.8 \\
\hline 30 & $17-05-2010$ & 03:06:03 & 77.328 & 35.023 & 92 & 3.2 & 0.45 & 3.2 & 1.7 \\
\hline 31 & $19-05-2010$ & 07:41:00 & 77.21 & 35.238 & 63.7 & 2.7 & 0.26 & 1.6 & 2.8 \\
\hline 32 & $20-05-2010$ & 04:06:09 & 77.387 & 34.954 & 2.7 & 2.6 & 0.12 & 1.9 & 2.9 \\
\hline 33 & 28-05-2010 & 05:45:42 & 77.248 & 35.1 & 11.1 & 3.1 & 0.47 & 1.1 & 3.6 \\
\hline 34 & 29-05-2010 & 08:32:09 & 77.308 & 35.018 & 6.5 & 2.8 & 0.38 & 2.5 & 5 \\
\hline 35 & $30-05-2010$ & $17: 24: 54$ & 77.425 & 35.045 & 5.7 & 3.9 & 0.78 & 3.8 & 1.3 \\
\hline 36 & 03-06-2010 & 02:51:40 & 77.2 & 34.89 & 4.3 & 2.7 & 0.24 & 4.7 & 1.9 \\
\hline 37 & 04-06-2010 & $22: 12: 18$ & 77.258 & 35.15 & 2.1 & 4.2 & 0.26 & 1.6 & 1.9 \\
\hline
\end{tabular}




\section{Continued}

\begin{tabular}{|c|c|c|c|c|c|c|c|c|c|}
\hline 38 & 06-06-2010 & 03:21:12 & 77.024 & 35.27 & 8.1 & 3.9 & 0.53 & 3.4 & 2.7 \\
\hline 39 & 08-06-2010 & $05: 45: 42$ & 77.226 & 35.34 & 9 & 3.8 & 0.45 & 4.3 & 3.6 \\
\hline 40 & 09-06-2010 & 08:32:09 & 77.119 & 35.147 & 45 & 4.2 & 0.47 & 2.8 & 3.4 \\
\hline 41 & 10-06-2010 & $17: 24: 54$ & 77.333 & 35.159 & 43 & 3.6 & 0.59 & 0.9 & 4.1 \\
\hline 42 & 23-06-2010 & 02:51:40 & 76.8 & 35.5 & 1.4 & 3.7 & 0.32 & 1 & 3.7 \\
\hline 43 & 24-06-2010 & $22: 12: 18$ & 77.795 & 35.4 & 12.3 & 2.5 & 0.26 & 2.7 & 1.8 \\
\hline 44 & $11-07-2010$ & 03:21:12 & 77.2 & 35.4 & 12.8 & 3.7 & 0.89 & 0.5 & 4.9 \\
\hline 45 & $12-07-2010$ & $13: 32: 13$ & 76.793 & 35.102 & 9.5 & 3.1 & 0.67 & 1.1 & 0.9 \\
\hline 46 & $16-07-2010$ & $21: 51: 17$ & 77.336 & 35.024 & 9.8 & 1.8 & 0.58 & 1.2 & 1.8 \\
\hline 47 & 21-07-2010 & $13: 26: 00$ & 77.466 & 35.016 & 0.1 & 3 & 0.12 & 3.4 & 4.6 \\
\hline 48 & $26-07-2010$ & 01:42:00 & 76.872 & 35.622 & 72 & 3.2 & 0.45 & 1.2 & 4.3 \\
\hline 49 & 28-07-2010 & 11:51:03 & 76.917 & 35.582 & 9.7 & 4.3 & 0.38 & 4.7 & 2.6 \\
\hline 50 & 01-08-2010 & 06:09:44 & 76.988 & 35.523 & 7.4 & 3 & 0.36 & 1.7 & 1.6 \\
\hline 51 & 03-08-2010 & 01:20:07 & 77.141 & 35.335 & 10 & 2.8 & 0.16 & 1.5 & 2.7 \\
\hline 52 & 06-08-2010 & $12: 19: 50$ & 77.18 & 35.287 & 1.7 & 4 & 0.23 & 0.8 & 1.9 \\
\hline 53 & 12-08-2010 & 03:06:03 & 78.594 & 34.19 & 6.7 & 2.5 & 0.39 & 3.7 & 4.3 \\
\hline 54 & $16-08-2010$ & 00:30:59 & 78.331 & 33.974 & 10 & 3.2 & 0.48 & 2.6 & 4.3 \\
\hline 55 & 18-08-2010 & $17: 24: 54$ & 77.298 & 35.177 & 12 & 3 & 0.59 & 1.2 & 4.9 \\
\hline 56 & 19-08-2010 & $14: 11: 29$ & 77.219 & 35.177 & 16.8 & 2.3 & 0.66 & 3.6 & 1.2 \\
\hline 57 & 24-08-2010 & $16: 25: 32$ & 71.282 & 36.49 & 10 & 3.15 & 0.38 & 1.8 & 3.2 \\
\hline 58 & 31-08-2010 & $20: 17: 10$ & 77.355 & 35.068 & 13.7 & 3.6 & 0.12 & 1.6 & 3.4 \\
\hline 59 & 02-09-2010 & $00: 21: 12$ & 77.044 & 35.519 & 14.5 & 2.6 & 0.49 & 4.3 & 4.9 \\
\hline 60 & 05-09-2010 & 04:15:47 & 77.591 & 34.969 & 71.2 & 2.2 & 0.27 & 1.7 & 3.7 \\
\hline 61 & 06-09-2010 & $23: 30: 15$ & 77.334 & 34.787 & 87.2 & 3.6 & 0.72 & 1.7 & 2.8 \\
\hline 62 & 07-09-2010 & $14: 36: 54$ & 77.431 & 34.75 & 15.4 & 2.4 & 0.45 & 3.2 & 1.7 \\
\hline 63 & 09-09-2010 & $16: 13: 12$ & 77.345 & 34.84 & 63.6 & 4.5 & 0.26 & 1.6 & 2.8 \\
\hline 64 & $10-09-2010$ & $13: 18: 15$ & 77.412 & 35.004 & 11.1 & 2.7 & 0.12 & 1.9 & 2.9 \\
\hline 65 & $15-09-2010$ & 21:21:32 & 77.452 & 34.76 & 26 & 2.8 & 0.47 & 1.1 & 3.6 \\
\hline 66 & 17-09-2010 & $14: 11: 16$ & 77.661 & 35.926 & 13.2 & 4.1 & 0.38 & 2.5 & 5 \\
\hline 67 & 19-09-2010 & $12: 43: 17$ & 71.478 & 36.931 & 0.1 & 3.5 & 0.36 & 3.2 & 2.7 \\
\hline 68 & 01-10-2010 & 02:00:27 & 77.158 & 34.652 & 10 & 3 & 0.78 & 3.8 & 1.3 \\
\hline 69 & 03-10-2010 & $10: 19: 57$ & 77.081 & 35.48 & 12 & 2.6 & 0.24 & 4.7 & 1.9 \\
\hline 70 & 09-10-2010 & 04:06:09 & 77.401 & 34.495 & 10 & 3.3 & 0.26 & 1.6 & 1.9 \\
\hline 71 & $11-10-2010$ & $05: 45: 42$ & 77.59 & 34.7 & 9 & 4 & 0.53 & 3.4 & 2.7 \\
\hline 72 & $13-10-2010$ & 08:32:09 & 77.085 & 35.495 & 6 & 2.8 & 0.45 & 4.3 & 3.6 \\
\hline 73 & $14-10-2010$ & $17: 24: 54$ & 77.156 & 35.366 & 10 & 2.1 & 0.47 & 2.8 & 3.4 \\
\hline 74 & $15-10-2010$ & 01:34:12 & 77.887 & 34.69 & 10 & 3 & 0.59 & 0.9 & 4.1 \\
\hline 75 & $18-10-2010$ & $11: 47: 21$ & 77.309 & 35.19 & 10 & 2.8 & 0.32 & 1 & 3.7 \\
\hline 76 & $22-10-2010$ & $12: 39: 42$ & 76.725 & 35.44 & 10.3 & 3.3 & 0.26 & 2.7 & 1.8 \\
\hline 77 & $26-10-2010$ & 15:33:18 & 76.786 & 35.334 & 37.7 & 2.8 & 0.89 & 0.5 & 4.9 \\
\hline 78 & 28-10-2010 & 01:16:29 & 77.21 & 35.24 & 3.2 & 2.8 & 0.67 & 1.1 & 0.9 \\
\hline
\end{tabular}




\section{Continued}

\begin{tabular}{|c|c|c|c|c|c|c|c|c|c|}
\hline 79 & 01-11-2010 & 04:03:11 & 77.125 & 35.427 & 5.9 & 3.2 & 0.58 & 1.2 & 1.8 \\
\hline 80 & 02-11-2010 & 10:01:16 & 77.58 & 34.671 & 4.9 & 2.3 & 0.12 & 3.4 & 4.6 \\
\hline 81 & 07-11-2010 & 07:15:18 & 77.552 & 34.805 & 6.2 & 3.9 & 0.45 & 1.2 & 4.3 \\
\hline 82 & 09-11-2010 & 01:42:18 & 70.746 & 35.732 & 10 & 3.6 & 0.38 & 4.7 & 2.6 \\
\hline 83 & $10-11-2010$ & $12: 55: 42$ & 77.556 & 34.721 & 83 & 2.6 & 0.36 & 1.7 & 1.6 \\
\hline 84 & 13-11-2010 & 19:49:00 & 77.537 & 35.362 & 4.6 & 3.4 & 0.16 & 1.5 & 2.7 \\
\hline 85 & $14-11-2010$ & 20:01:56 & 77.367 & 35.042 & 8.2 & 3.7 & 0.23 & 0.8 & 1.9 \\
\hline 86 & $15-11-2010$ & 07:05:18 & 77.619 & 34.586 & 10 & 3.9 & 0.39 & 3.7 & 4.3 \\
\hline 87 & $02-12-2010$ & 01:42:18 & 74.246 & 35.249 & 10 & 3 & 0.48 & 2.6 & 4.3 \\
\hline 88 & 03-12-2010 & $12: 55: 00$ & 76.802 & 34.023 & 2 & 2.54 & 0.59 & 1.2 & 4.9 \\
\hline 89 & $06-12-2010$ & 02:55:03 & 77.54 & 35.011 & 0.1 & 3.5 & 0.66 & 3.6 & 1.2 \\
\hline 90 & 09-12-2010 & 07:29:27 & 77.412 & 35.1 & 9.8 & 3.8 & 0.38 & 1.8 & 3.2 \\
\hline 91 & $18-12-2010$ & 20:01:20 & 77.153 & 35.326 & 10 & 2.6 & 0.12 & 1.6 & 3.4 \\
\hline 92 & $19-12-2010$ & 04:18:17 & 76.692 & 35.109 & 10 & 3.3 & 0.49 & 4.3 & 4.9 \\
\hline 93 & 01-01-2011 & $10: 15: 47$ & 77.29 & 34.795 & 9.4 & 2.2 & 0.27 & 1.7 & 3.7 \\
\hline 94 & 20-01-2011 & $22: 43: 56$ & 77.552 & 34.853 & 10 & 4.2 & 0.4 & 4.7 & 3.7 \\
\hline 95 & 08-02-2011 & $16: 52: 59$ & 77.43 & 35.398 & 132.4 & 3.2 & 0.21 & 5 & 1.9 \\
\hline 96 & $13-02-2011$ & 11:52:19 & 77.195 & 35.408 & 10 & 2 & 0.36 & 3.2 & 2.7 \\
\hline 97 & $18-05-2011$ & 10:19:57 & 77.177 & 35.224 & & 71.2 & 0.47 & 4.3 & 1.2 \\
\hline 98 & 05-07-2011 & 04:06:09 & 77.29 & 35.14 & 9.8 & 2.2 & 0.58 & 2.4 & 3.9 \\
\hline 99 & 05-07-2011 & $05: 45: 42$ & 77.517 & 34.892 & 10 & 3.7 & 0.39 & 4.6 & 3.8 \\
\hline 100 & $10-07-2011$ & 08:32:09 & 77.11 & 35.4 & 12 & 3 & 0.89 & 1.7 & 3.4 \\
\hline 101 & 24-07-2011 & $17: 24: 54$ & 77.09 & 35.21 & 11 & 4.2 & 0.76 & 3.4 & 2.6 \\
\hline 102 & 04-09-2011 & $14: 11: 29$ & 77.049 & 34.843 & 10 & 2.2 & 0.63 & 5 & 1.7 \\
\hline 103 & 05-09-2011 & $16: 25: 32$ & 77.208 & 34.911 & 15 & 1.7 & 0.42 & 1.2 & 3.6 \\
\hline 104 & 06-09-2011 & $20: 17: 10$ & 77.071 & 34.815 & 10 & 2.2 & 0.53 & 4.3 & 4.9 \\
\hline 105 & 25-09-2011 & $06: 47: 49$ & 76.679 & 35.39 & 10 & 3.1 & 0.56 & 5 & 3.7 \\
\hline 106 & $16-10-2011$ & 00:31:18 & 77.937 & 34.702 & 14 & 2.3 & 0.39 & 4.8 & 1.7 \\
\hline 107 & $24-10-2011$ & 10:17:10 & 77.24 & 35.12 & 57 & 2.6 & 0.57 & 5.2 & 3.5 \\
\hline 108 & $29-10-2011$ & 20:53:29 & 76.598 & 35.298 & 45 & 1.7 & 0.17 & 3.7 & 2.9 \\
\hline 109 & 01-11-2011 & 18:37:00 & 77.863 & 34.563 & 10 & 3.5 & 0.34 & 4.9 & 2.6 \\
\hline 110 & 02-11-2011 & $15: 18: 44$ & 76.761 & 35.135 & 16.8 & 1.7 & 0.12 & 4.3 & 3.8 \\
\hline 111 & 02-11-2011 & 03:21:12 & 77.211 & 35.132 & 4.3 & 2.8 & 0.26 & 2.9 & 3.1 \\
\hline 112 & 06-11-2011 & $11: 26: 54$ & 77.244 & 35.247 & 55.9 & 3 & 0.69 & 1.3 & 4.9 \\
\hline 113 & $11-11-2011$ & 03:19:39 & 77.228 & 35.133 & 1.2 & 4.2 & 0.34 & 3.7 & 1.9 \\
\hline 114 & $12-11-2011$ & 01:42:51 & 76.779 & 35.405 & 47 & 2 & 0.43 & 2.6 & 2.1 \\
\hline 115 & $12-11-2011$ & 11:51:03 & 77.412 & 35.332 & 42 & 3.6 & 0.56 & 1.8 & 2.6 \\
\hline 116 & 17-11-2011 & $20: 17: 10$ & 77.082 & 35.038 & 2.9 & 2.6 & 0.71 & 2.8 & 1.9 \\
\hline 117 & 21-11-2011 & 00:03:00 & 76.621 & 35.303 & 45 & 1.8 & 0.63 & 4.3 & 3.2 \\
\hline 118 & 27-11-2011 & 05:27:00 & 77.433 & 34.83 & 110 & 2.1 & 0.59 & 3.1 & 2.7 \\
\hline
\end{tabular}


R. Parshad et al.

\section{Continued}

\begin{tabular}{|c|c|c|c|c|c|c|c|c|c|}
\hline 119 & 30-11-2011 & 00:34:00 & 77.997 & 34.862 & 10 & 1.9 & 0.89 & 1.7 & 3.9 \\
\hline 120 & 02-12-2011 & 10:19:37 & 77.007 & 35.12 & 9.5 & 3.8 & 0.63 & 2.6 & 3.2 \\
\hline 121 & 08-12-2011 & 14:06:09 & 77.82 & 35.18 & 4.3 & 2.7 & 0.39 & 3.7 & 4.3 \\
\hline 122 & 09-12-2011 & $11: 45: 42$ & 77.955 & 35.58 & 97.8 & 3.2 & 0.48 & 2.6 & 4.3 \\
\hline 123 & $12-12-2011$ & 08:32:09 & 77.173 & 35.259 & 10 & 3.2 & 0.59 & 1.2 & 4.9 \\
\hline 124 & $14-12-2011$ & $17: 24: 54$ & 77.167 & 35.412 & 49.5 & 3 & 0.66 & 3.6 & 1.2 \\
\hline 125 & $29-12-2011$ & 02:51:40 & 77.15 & 35.36 & 63.7 & 2.4 & 0.38 & 1.8 & 3.2 \\
\hline 126 & $30-12-2011$ & $22: 12: 18$ & 77.183 & 35.252 & 1.9 & 4.3 & 0.12 & 1.6 & 3.4 \\
\hline 127 & $18-02-2012$ & 03:21:12 & 77.233 & 35.14 & 10 & 2.6 & 0.49 & 4.3 & 4.9 \\
\hline 128 & 23-02-2012 & 11:26:00 & 77.345 & 35.084 & 10 & 3.9 & 0.27 & 1.7 & 3.7 \\
\hline 129 & 02-03-2012 & 03:19:00 & 77.424 & 34.935 & 100.7 & 3 & 0.4 & 4.7 & 3.7 \\
\hline 130 & 29-03-2012 & 01:42:00 & 77.558 & 34.711 & 125 & 2.1 & 0.21 & 5 & 1.9 \\
\hline 131 & 09-04-2012 & 11:51:03 & 77.581 & 34.681 & 10 & 2.8 & 0.36 & 3.2 & 2.7 \\
\hline 132 & 18-04-2012 & 06:09:44 & 76.95 & 35.481 & 2.7 & 3.9 & 0.47 & 4.3 & 1.2 \\
\hline 133 & $19-04-2012$ & 01:20:07 & 77.088 & 35.382 & 10 & 2.7 & 0.58 & 2.4 & 3.9 \\
\hline 134 & 20-04-2012 & $12: 19: 50$ & 77.166 & 35.196 & 12 & 2 & 0.39 & 4.6 & 3.8 \\
\hline 135 & 21-04-2012 & 03:07:23 & 77.257 & 35.048 & 13.7 & 3.8 & 0.89 & 1.7 & 3.4 \\
\hline 136 & 27-04-2012 & 20:01:56 & 77.34 & 34.899 & 3.2 & 4 & 0.76 & 3.4 & 2.6 \\
\hline 137 & 28-04-2012 & 07:05:18 & 77.261 & 35.153 & 10 & 3.8 & 0.63 & 5 & 1.7 \\
\hline 138 & 01-05-2012 & 01:42:18 & 77.286 & 35.114 & 7.8 & 2.6 & 0.42 & 1.2 & 3.6 \\
\hline 139 & 07-05-2012 & $12: 55: 00$ & 77.23 & 35.161 & 3.8 & 4.4 & 0.53 & 4.3 & 4.9 \\
\hline 140 & $11-05-2012$ & 10:38:12 & 77.257 & 35.121 & 10 & 2.5 & 0.56 & 5 & 3.7 \\
\hline 141 & $13-05-2012$ & 22:36:41 & 77.317 & 35.099 & 8.7 & 3.4 & 0.39 & 4.8 & 1.7 \\
\hline 142 & $15-05-2012$ & $12: 21: 44$ & 76.891 & 35.621 & 9.4 & 3.8 & 0.57 & 5.2 & 3.5 \\
\hline 143 & $16-05-2012$ & 09:43:07 & 77.016 & 35.564 & 49.8 & 3.7 & 0.17 & 3.7 & 2.9 \\
\hline 144 & $16-05-2012$ & 00:57:09 & 77.054 & 35.472 & 43.7 & 4.1 & 0.34 & 4.9 & 2.6 \\
\hline 145 & $17-05-2012$ & $18: 22: 54$ & 77.122 & 35.402 & 90.2 & 2.9 & 0.12 & 4.3 & 3.8 \\
\hline 146 & $18-05-2012$ & 03:40:40 & 77.163 & 35.292 & 48.4 & 3 & 0.26 & 2.9 & 3.1 \\
\hline 147 & 20-05-2012 & 11:18:39 & 77.19 & 35.212 & 10 & 4.2 & 0.69 & 1.3 & 4.9 \\
\hline 148 & 21-05-2012 & $17: 26: 41$ & 77.209 & 35.317 & 53.8 & 2 & 0.34 & 3.7 & 1.9 \\
\hline 149 & 27-05-2012 & 01:08:29 & 77.252 & 35.236 & 9.4 & 2.3 & 0.43 & 2.6 & 2.1 \\
\hline 150 & 27-05-2012 & $17: 46: 41$ & 77.344 & 35.101 & 8.4 & 3 & 0.56 & 1.8 & 2.6 \\
\hline 151 & 28-05-2012 & 00:38:12 & 77.123 & 35.378 & 59.2 & 3 & 0.71 & 2.8 & 1.9 \\
\hline 152 & 29-05-2012 & $12: 36: 41$ & 77.218 & 35.118 & 4 & 3.6 & 0.63 & 4.3 & 3.2 \\
\hline 153 & $30-05-2012$ & 10:19:57 & 76.927 & 35.653 & 12.1 & 4.3 & 0.59 & 3.1 & 2.7 \\
\hline 154 & 04-06-2012 & 04:06:09 & 77.318 & 35.131 & 10 & 3.4 & 0.12 & 1.9 & 2.9 \\
\hline 155 & 05-06-2012 & $05: 45: 42$ & 76.887 & 35.635 & 61.7 & 3.7 & 0.47 & 1.1 & 3.6 \\
\hline 156 & 08-06-2012 & 08:32:09 & 77.407 & 35.021 & 67.9 & 3.2 & 0.38 & 2.5 & 5 \\
\hline 157 & $11-06-2012$ & $17: 24: 54$ & 77.186 & 35.139 & 10 & 3.9 & 0.78 & 3.8 & 1.3 \\
\hline 158 & $12-06-2012$ & $02: 51: 40$ & 77.322 & 35.102 & 71.3 & 2.9 & 0.24 & 4.7 & 1.9 \\
\hline
\end{tabular}




\section{Continued}

\begin{tabular}{|c|c|c|c|c|c|c|c|c|c|}
\hline 159 & 14-06-2012 & $22: 12: 18$ & 77.325 & 34.986 & 7.4 & 2.4 & 0.26 & 1.6 & 1.9 \\
\hline 160 & 15-06-2012 & 03:21:12 & 77.226 & 35.282 & 5.2 & 3.6 & 0.53 & 3.4 & 2.7 \\
\hline 161 & 20-06-2012 & $11: 26: 00$ & 77.282 & 34.744 & 12.8 & 3.8 & 0.45 & 4.3 & 3.6 \\
\hline 162 & 22-06-2012 & 03:19:00 & 77.611 & 34.577 & 45.8 & 3.2 & 0.47 & 2.8 & 3.4 \\
\hline 163 & 24-06-2012 & 01:42:00 & 77.419 & 34.641 & 94.3 & 3.5 & 0.59 & 0.9 & 4.1 \\
\hline 164 & 29-06-2012 & 11:51:03 & 77.557 & 34.776 & 68.5 & 2.3 & 0.32 & 1 & 3.7 \\
\hline 165 & 11-07-2012 & 06:09:44 & 77.481 & 34.86 & 10 & 2.8 & 0.26 & 2.7 & 1.8 \\
\hline 166 & 14-07-2012 & 01:20:07 & 77.545 & 34.703 & 74.9 & 4.2 & 0.89 & 0.5 & 4.9 \\
\hline 167 & 17-07-2012 & $12: 19: 50$ & 77.007 & 35.494 & 6.8 & 3.7 & 0.67 & 1.1 & 0.9 \\
\hline 168 & 18-07-2012 & 03:07:23 & 77.134 & 35.388 & 0.9 & 2.1 & 0.58 & 1.2 & 1.8 \\
\hline 169 & 23-07-2012 & 20:01:56 & 77.361 & 35 & 10 & 2.3 & 0.12 & 3.4 & 4.6 \\
\hline 170 & 31-07-2012 & 07:05:18 & 77.634 & 34.637 & 47.9 & 3.8 & 0.45 & 1.2 & 4.3 \\
\hline 171 & 01-08-2012 & 01:42:18 & 77.509 & 34.748 & 110.1 & 3.5 & 0.38 & 4.7 & 2.6 \\
\hline 172 & 06-08-2012 & $12: 55: 00$ & 77.47 & 34.691 & 320.1 & 2.9 & 0.36 & 1.7 & 1.6 \\
\hline 173 & 12-08-2012 & 07:31:12 & 77.204 & 34.847 & 10 & 3.7 & 0.16 & 1.5 & 2.7 \\
\hline 174 & 17-08-2012 & 10:38:12 & 77.504 & 35.149 & 10.7 & 3.6 & 0.23 & 0.8 & 1.9 \\
\hline 175 & 26-08-2012 & $22: 36: 41$ & 76.954 & 35.243 & 8.4 & 2.3 & 0.39 & 3.7 & 4.3 \\
\hline 176 & 28-08-2012 & $12: 21: 44$ & 77.072 & 35.458 & 10.2 & 3.4 & 0.48 & 2.6 & 4.3 \\
\hline 177 & 01-09-2012 & 09:43:07 & 77.388 & 34.966 & 12 & 3.6 & 0.59 & 1.2 & 4.9 \\
\hline 178 & 02-09-2012 & 00:57:09 & 77.01 & 35.256 & 74 & 3.2 & 0.66 & 3.6 & 1.2 \\
\hline 179 & 05-09-2012 & $18: 22: 54$ & 77.146 & 35.255 & 86 & 2.8 & 0.38 & 1.8 & 3.2 \\
\hline 180 & 05-09-2012 & 03:40:40 & 77.254 & 35.193 & 73.7 & 3.9 & 0.12 & 1.6 & 3.4 \\
\hline 181 & 09-09-2012 & 11:18:39 & 77.255 & 35.245 & 98.7 & 4.1 & 0.69 & 1.3 & 4.9 \\
\hline 182 & 10-09-2012 & $17: 26: 41$ & 77.633 & 34.654 & 12 & 4 & 0.34 & 3.7 & 1.9 \\
\hline 183 & 07-10-2012 & 01:08:29 & 77.477 & 34.911 & 10 & 2.6 & 0.43 & 2.6 & 2.1 \\
\hline 184 & 08-10-2012 & $17: 46: 41$ & 76.629 & 35.46 & 10 & 1.9 & 0.56 & 1.8 & 2.6 \\
\hline 185 & 09-10-2012 & 00:38:12 & 77.241 & 35.402 & 10 & 1.3 & 0.71 & 2.8 & 1.9 \\
\hline 186 & $15-10-2012$ & $12: 36: 41$ & 77.121 & 35.12 & & 12 & 0.63 & 4.3 & 3.2 \\
\hline 187 & 15-10-2012 & 02:49:39 & 77.464 & 34.918 & 10 & 6.9 & 0.59 & 3.1 & 2.7 \\
\hline 188 & 20-10-2012 & 07:31:12 & 77.429 & 34.893 & 10 & 2.6 & 0.89 & 1.7 & 3.9 \\
\hline 189 & 25-10-2012 & 10:38:12 & 77.53 & 35.14 & 47.9 & 3.5 & 0.63 & 2.6 & 3.2 \\
\hline 190 & 26-10-2012 & $22: 36: 41$ & 77.21 & 35.12 & 12.9 & 3.2 & 0.39 & 3.7 & 4.3 \\
\hline 191 & 06-11-2012 & $12: 21: 44$ & 77.198 & 34.916 & 432.8 & 2.9 & 0.48 & 2.6 & 4.3 \\
\hline 192 & 09-11-2012 & 09:43:07 & 77.483 & 34.922 & 134 & 2.8 & 0.59 & 1.2 & 4.9 \\
\hline 193 & 16-11-2012 & 00:57:09 & 76.914 & 34.975 & 10 & 2.7 & 0.66 & 3.6 & 1.2 \\
\hline 194 & 23-11-2012 & $18: 22: 54$ & 77.406 & 34.9 & 10 & 2.1 & 0.38 & 1.8 & 3.2 \\
\hline 195 & 28-11-2012 & 03:40:40 & 77.222 & 34.912 & 172 & 2.2 & 0.12 & 1.6 & 3.4 \\
\hline 196 & 05-12-2012 & $19: 43: 07$ & 76.917 & 35.12 & 218.9 & 3.9 & 0.49 & 4.3 & 4.9 \\
\hline 197 & 24-12-2012 & $22: 49: 18$ & 77.945 & 34.93 & 115.6 & 3 & 0.27 & 1.7 & 3.7 \\
\hline 198 & 25-12-2012 & $10: 49: 38$ & 77.684 & 35.238 & 126.7 & 2.8 & 0.4 & 4.7 & 3.7 \\
\hline
\end{tabular}


region, India and consistent with the geological section of the area prepared by [24] (Figure 6(a)).

Considerable amount of broken ice chunks were observed near the snout of siachen glacier during field visit in Aug'2011 (Figure 7). The field personnel deployed at field stations reported that the cracking mainly observed contemporaneous to the shacking. The close association of log book maintained at field sites for keeping

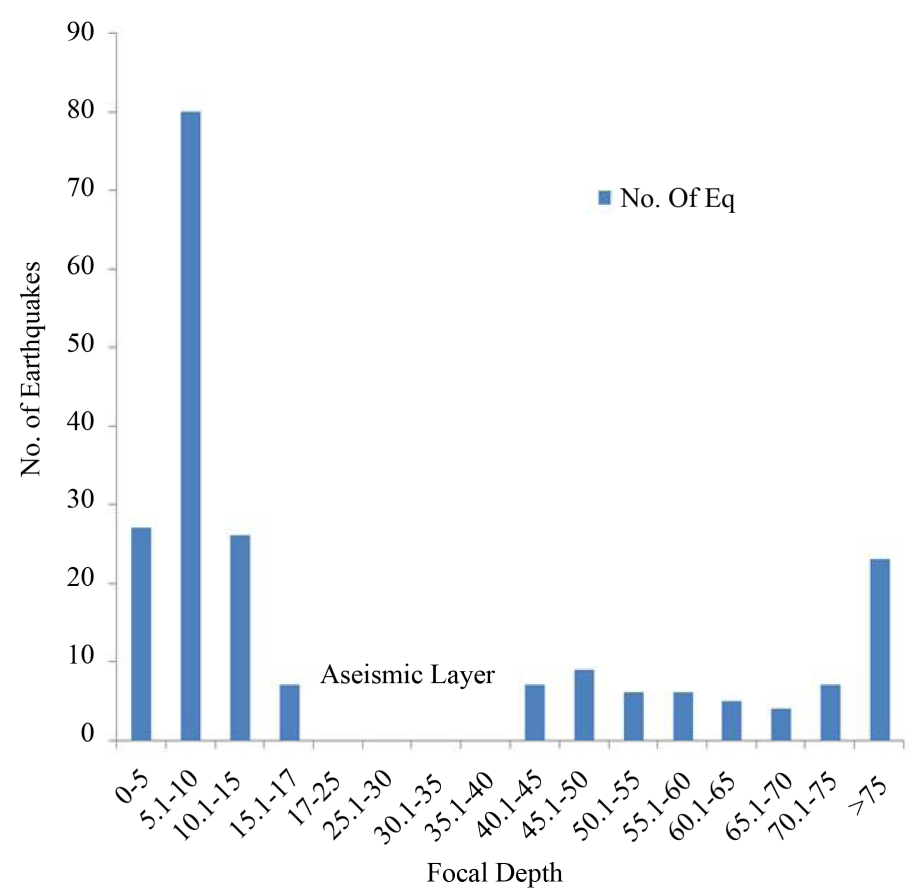

Figure 5. Plot of number of earthquakes versus focal depth.

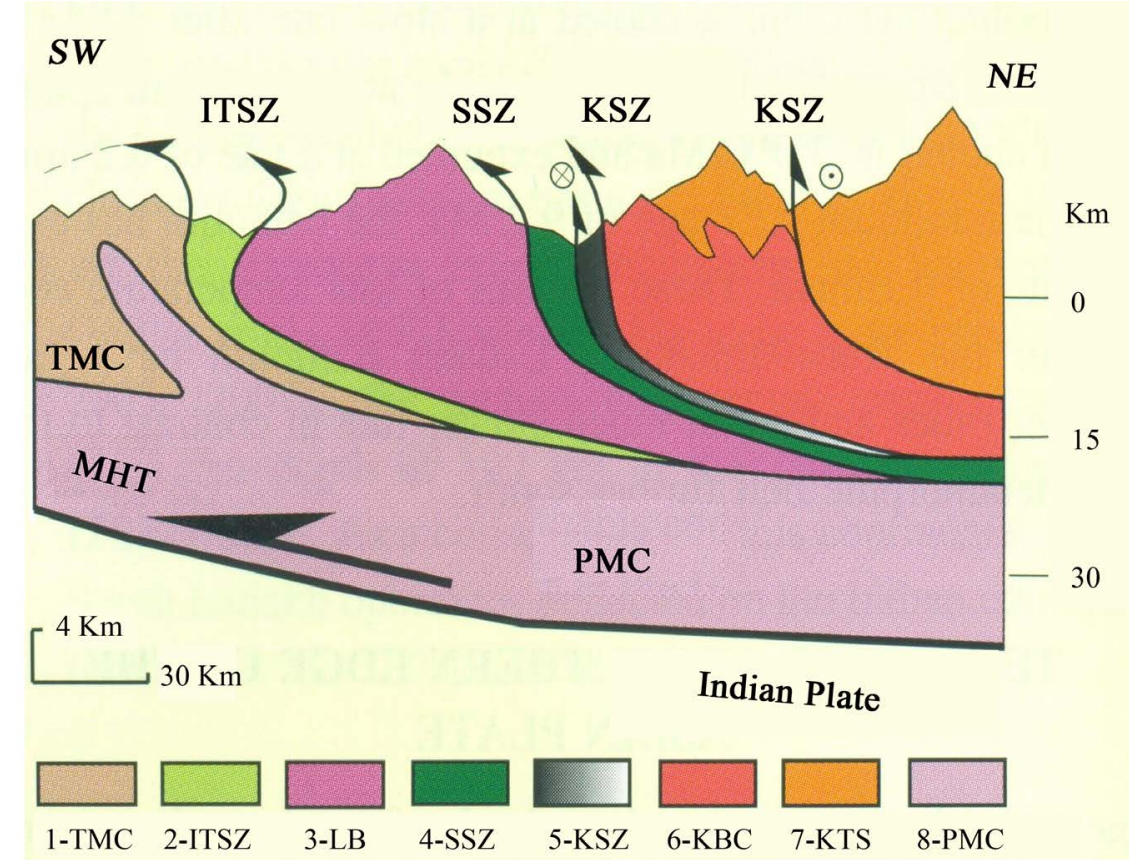

(a)

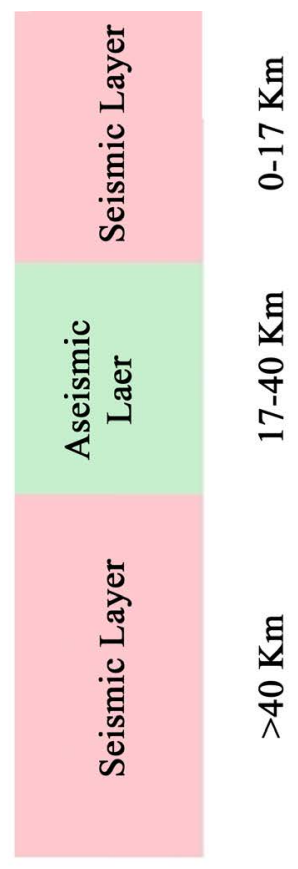

(b)

Figure 6. (a) Geological cross-section through the Northern parts of NW Himalaya and Karakorum, after [16]; (b) Aseismic layer in the mid-crust. 

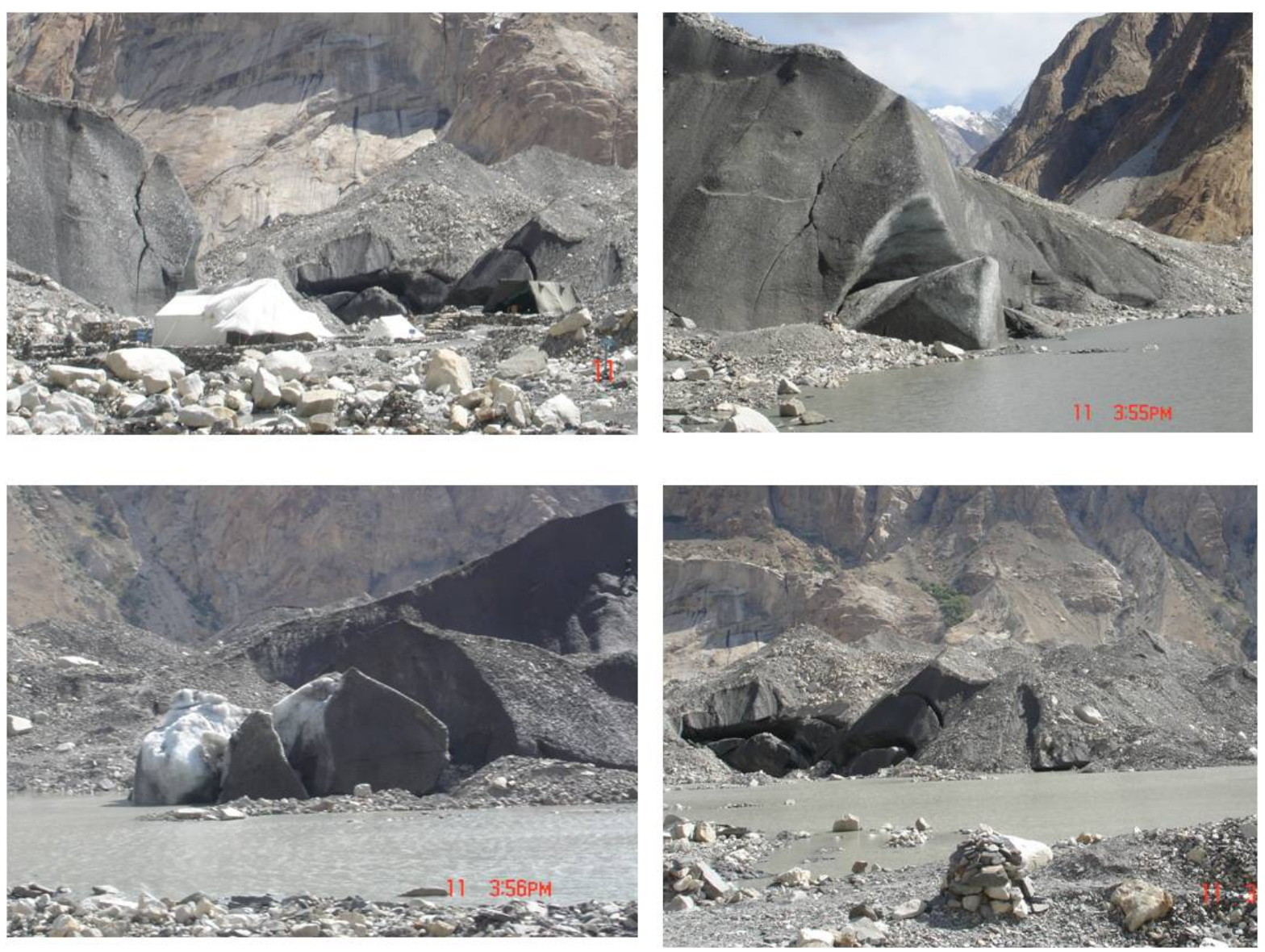

Figure 7. Field Photographs showing cracking in the snout region.

record of shaking and seismic data recorded at field seismic stations indicates that these cracking mainly associated with tremors occurring near the snout of the siachen glacier. Moreover few earthquake events are located in the snout region of glacier it is evident that these earthquake events are responsible for cracking in the snout of glacier. As the Karakoram fault passes through the main body of siachen glacier as well as through snout region of siachen glacier (Figure 1) such tremors will keep occurring due to the presence of this active fault and theses tremors may have very serious implication on the snout of the siachen glacier.

\section{Conclusion}

Aseismic layer $(17-40 \mathrm{Km})$ is detected in the mid-crust of Nubra-Siachen region of NW, Himalaya with the help of locally observed seismic data. This layer is sandwiched between two seismically active layers. This aseismic layer shows the good spatial correlation with the low resistivity layer [26]. Results of this study support the proposed "partially melted crust for this region" by [24]. The observed local seismicity for the period Jan' 2010-Dec' 2012 in Siachen-Nubra region of Jammu \& Kashmir is mainly oriented along the Karakoram Fault and clustered in Shyok suture zone \& Karakoram Shear zone. The moment magnitudes of these events lie between 1.3 and 4.3. The depth distribution of earthquake events shows that most of the local earthquakes in this region have been occurred at upper part of the crust. Such shallow seismic activity in the glacier region may affect the glaciers. These aspects need to be studied in more detail.

\section{Acknowledgements}

The research was funded by the MoES grant no. MoES/P.). (Seismo)/1(83)/2010, dated 10/08/2010. The support by SASE personnel in field data collection is highly acknowledged. 


\section{References}

[1] Ni, J. and Barazangi, M. (1984) Seismotectonics of the Himalayan Collision Zone Geometry of the Under Thrusting Indian Plate Beneath The Himalaya. Journal of Geophysical Research, 89, 1147-1163. http://dx.doi.org/10.1029/JB089iB02p01147

[2] Seeber, L. and Armbruster, J.G. (1981) Great Detachment Earthquakes along the Himalayan Arc and Long-Term Forecasting. In: Earthquake Prediction: An Int. Review, Am. Geophys. Union (Maurice Ewing Series), 4, 259-277. http://dx.doi.org/10.1029/me004p0259

[3] Valdiya, K.S. (1980) The Two Intracrustal Boundary Thrusts of the Himalaya. Tectnophysics, 66, 323-348. http://dx.doi.org/10.1016/0040-1951(80)90248-6

[4] SASE, Annual Technical Report 2002-2003 (2003) (Unpublished).

[5] Yin, A. and Harrison, T.M. (2000) Geologic Evolution of Himalayan-Tibetan Orogen. Annual Review of Earth and Planetary Sciences, 28, 211-280. http://dx.doi.org/10.1146/annurev.earth.28.1.211

[6] Wang, Q., et al. (2001) Present-Day Crustal Deformation in China Constrained by Global Positioning System Measurements. Science, 294, 574-577.

[7] Gansser, A. (1964) Geology of the Himalayas. Willey Interscience, London.

[8] Rai, S.S., Priestley, K. and Gaur, V.K. (2006) Configuration of the Indian Moho beneath the NW Himalaya and Ladakh. Geophysical Research Letters, 33, L15308. http://dx.doi.org/10.1029/2006gl026076

[9] Rai, H. (1983) Geology of the Nubra Valley and Its Significance on the Evolution of the Ladakh Himalaya, Geology of Indus Suture Zone of Ladakh. In: Thakur, V.C. and Sharma, K.K., Eds., Geology of Indus Suture Zone of Ladakh, WIHG, Dehradun, 79-97.

[10] Chevalier, M.L., Ryerson, F.J., Tapponnier, P., Finkel, R.C., Van Der Woerd, J., Haibing, L. and Qing, L. (2005) SlipRate Measurements on the Karakoram Fault May Imply Secular Variations in Fault Motion. Science, 307, 411-414. http://dx.doi.org/10.1126/science.1105466

[11] Dunlap, W.J., Weinberg, R.F. and Searle, M.P. (1998) Karakoram Fault Zone Rocks Cool in Two Phases. Journal of the Geological Society, 155, 903-912. http://dx.doi.org/10.1144/gsjgs.155.6.0903

[12] Searle, M.P., Dewey, J.F., Dunlap, W.J., Strachan, R.A. and Weinberg, R.F. (1998) Transpressional Tectonics along the Karakoram Fault Zone, Northern Ladakh: Constraints Ontibetan Extrusion. Geological Society, London, Special Publications, 135, 307-326. http://dx.doi.org/10.1144/GSL.SP.1998.135.01.20

[13] Searle, M.P. and Richard, J.P. (2007) Relationships between Right-Lateral Shear along the Karakoram Fault and Metamorphism, Magmatism, Exhumation and Uplift: Evidence from the K2-Gasherbrum, Pangong Ranges, North Pakistan and Ladakh. Journal of the Geological Society, 164, 439-450. http://dx.doi.org/10.1144/0016-76492006-072

[14] Phillips, R.J., Parrish, R.R. and Searle, M.P. (2004) Age Constraints on Ductile Deformation and Long-Term Slip Rated along the Karakoram Fault Zone, Ladakh. Earth and Planetary Science Letters, 226, 305-319. http://dx.doi.org/10.1016/j.epsl.2004.07.037

[15] Phillips, R.J. (2008) Geological Map of the Karakoram Fault Zone, Eastern Karakoram, Ladakh, NW Himalaya. Journal of Maps, 4, 21-37. http://dx.doi.org/10.4113/jom.2008.98

[16] Jain, A.K. and Singh, S. (2009) Geology and Tectonics of the Southeatern Ladakh and Karakoram. Geological Society of India, Bangalore.

[17] IS (2002) IS 1893-2002 (Part L) Indian Standard Criteria for Earthquake Resistant Design of Structures, Part 1 - General Provisions and Buildings. Bureau of Indian Standards, New Delhi.

[18] Dasgupta, S., Narula, P.L., Acharya, S.K. and Banerjee, J. (2000) Seismotectonicatlas of India and Its Environs. Geological Survey of India, Kolkata.

[19] SASE, Unpublished Reports.

[20] Pers. Communications with Localities during the Field Visit, 2011.

[21] Kumar, A., Kumar, A., Gupta, S.C., Jindal, A.K. and Ghangas, V. (2014) Seismicity and Source Parameters of Local Earthquakes in Bilaspur Region of Himachal Lesser Himalaya. Arabian Journal of Geosciences, 7, 2257-2267. http://dx.doi.org/10.1007/s12517-013-0929-y

[22] Kumar, A. (2011) Study of Earthquake Source Parameters Using Microearthquakes and Strong Motion Data. PhD Thesis, Indian Institute of Technology, Roorkee.

[23] Caldwell, W.B., Klemperer, S.L., Rai, S.S. and Lawrence, J.F. (2009) Partial Melt in the Upper-Middle Crust of the Northwest Himalaya Revealed by Rayleigh Wave Dispersion. Tectonophysics, 477, 58-65.

http://dx.doi.org/10.1016/j.tecto.2009.01.013 
[24] Lienert, B.R. (1994) Hypocenter 3.2: A Computer Program for Locating Earthquakes Locally Regionally and Globally. Hawai'i Institute of Geophysics and Planetology, Honolulu.

[25] Hanks, T.C. and Kanamori, H. (1979) A Moment Magnitude Scale. Journal of Geophysical Research, 84, $2348-2350$. http://dx.doi.org/10.1029/JB084iB05p02348

[26] Arora, B.R., Unsworth, M.J. and Rawat, G. (2007) Deep Resistivity Structure of the Northwest Indian Himalaya and Its Tectonic Implications. Geophysical Research Letters, 34, L04307. http://dx.doi.org/10.1029/2006gl029165 